Results and discussion: Literature review using the terms 'head and neck', 'cutaneous SCC', 'prognosis' and 'AJCC' yielded over 200 results pertaining to the prognostic features of HNcSCC, with fewer than 20 peer-reviewed, indexed manuscripts directly relevant to the prognostic performance of the AJCC8 HNcSCC staging system.

The evidence suggests that the omission of prognostically important characteristics (such as patient immunosuppression, depth of invasion, perineural invasion, extent of extranodal extension, and soft-tissue metastases) may contribute to the poor predictive performance of the staging system.

The questionnaire is being currently circulated and the Results will be provided.

\section{RETROTRANSPOSON ACTIVITY IN YOUNG PATIENTS WITH ORAL SQUAMOUS CELL CARCINOMA (OSCC)}

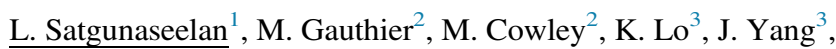
J. Clark ${ }^{4,5}$, R. Gupta ${ }^{1,4,5}$

${ }^{1}$ Department of Tissue Pathology and Diagnostic Oncology, Royal Prince Alfred Hospital, Sydney, Australia; ${ }^{2}$ Children's Cancer Institute Australia, Sydney, Australia; ${ }^{3}$ Sydney Bioinformatics and Biometrics, School of Mathematics and Statistics, University of Sydney, Sydney, Australia; ${ }^{4}$ Central Clinical School, University of Sydney, Sydney, Australia; and ${ }^{5}$ Sydney Head and Neck Cancer Institute, Chris O'Brien Lifehouse, Sydney, Australia

Background: A recent alarming increase in the incidence of OSCC has been seen in young patients in the absence of tobacco or alcohol use, or HPV integration. Retrotransposons are mobile DNA elements which have been shown to have a role in cancer, particularly LINE-1, with transposition events resulting in mutagenesis.

Aims: To compare the activity of three retrotransposon families (LINE-1, SVA and Alu) between young $(<45)$ and older patients with OSCC.

Methods: 7 young and 16 older non-HPV OSCC patients, whose genomes were sequenced at high depth, were identified from The Cancer Genome Atlas (TCGA). The Mobile Element Locator Tool (MELT) was employed to detect non-reference retrotransposon events in the selected genomes.

Results and conclusions: The total count of putative somatic LINE-1 insertions was significantly higher in the young as compared to the older cohort $(p=0.004)$. A significantly higher number of these events were found to occur close to genes $(p=0.019)$, with possible roles in cancer predisposition syndromes (e.g., OR4C45 and DTNB). RNA-Seq data is being analysed to assess the potential impact of the insertions on gene expression. The presence of elevated somatic LINE-1 activity in young patients may shed light on the increasing development of OSCC at an early age.

\section{HPV-RELATED MULTIPHENOTYPIC SINONASAL CARCINOMA, AN EMERGING ENTITY IN AN UNUSUAL LOCATION: A CASE REPORT}

C. Y. Chow, S. Sathiyamoorthy

Department of Anatomical Pathology, Division of Pathology, Singapore General Hospital, Singapore
Background: Human papillomavirus (HPV)-related multiphenotypic sinonasal carcinoma (HMSC), originally known as HPV-related carcinoma with adenoid cystic carcinoma-like features, is a distinct tumour recently described in the sinonasal tract. This peculiar tumour exhibits morphologic features of a salivary gland tumour including admixed ductal and myoepithelial elements, as well as displays an unusual pattern of surface involvement with atypical squamous cells. It is associated with high-risk HPV. Most cases usually present as a large and destructive sinonasal mass with high-grade histologic features. Aim: We present a case report of HMSC arising in the floor of mouth of a 57-year-old Chinese man.

Methods (clinical history): The patient was an ex-smoker who presented with a floor of mouth ulcer. The lesion was biopsy and was called a submucosal adenoid cystic carcinoma. Subsequently, the patient underwent wide resection of floor of mouth tumour, marginal mandibulectomy and neck dissection.

Results (pathological findings): Macroscopic examination revealed an ulcerative tumour measuring $2.5 \mathrm{~cm}$, extending to the margins. Microscopically, the tumour was composed of basaloid cells showing variable growth patterns including a cribriforming pattern that closely resembles adenoid cystic carcinoma. It was continuous with the mucosa that showed dysplastic squamous epithelium. On immunohistochemistry, the myoepithelial cells were positive for the myoepithelial markers. p16 was positive in both squamous and epithelial components. Metastatic carcinoma was present in two of the harvested lymph nodes. The patient developed pulmonary and liver metastases after two years of follow-up, which were confirmed histologically.

Conclusions: This is the first case of HMSC reported in the floor of mouth, which behaves more aggressively as opposed to those arise from the sinonasal tract. It is important to recognise HMSC as they have a different clinical behaviour. The clue to the diagnosis is recognising the atypical squamous cells on the surface, corroborated by 16 positivity in tumour cells.

\section{THE ROLE OF LIVER BIOPSY IN THE MANAGEMENT OF GRAFT DYSFUNCTION AT THE AUSTRALIAN NATIONAL TRANSPLANT UNIT (ANLTU): A RETROSPECTIVE STUDY OF 77 CASES}

\author{
M. Eghtedari ${ }^{1}$, C. McKenzie ${ }^{1,2}$, J. Kench ${ }^{1,2}$ \\ ${ }^{1}$ Central Clinical School, The University of Sydney School of \\ Medicine, Faculty of Medicine and Health, Australia; and \\ ${ }^{2}$ Department Tissue Pathology and Diagnostic Oncology, Royal \\ Prince Alfred Hospital, Sydney, Australia
}

Background: Clinically liver allograft rejection in liver transplant (LT) presents with non-specific findings such as fever, jaundice and elevated liver function tests (LFTs). Histopathological assessment of liver biopsies is currently the gold standard for diagnosing graft rejection. Liver biopsy can be associated with complications such as bleeding and sepsis.

Aim: To evaluate the role of liver biopsies in the diagnosis and management of liver rejection in patients who underwent LT at ANLTU in 2015 including (1) histological diagnoses of biopsies performed within 12-months post-LT; (2) changes in LFTs including alanine aminotransferase (ALT), gamma-glutamyl transpeptidase (GGT) and alkaline phosphatase (ALP) pre-and post-biopsy; and (3) changes to management pre-and postbiopsy. 